\title{
Effect of non-uniform magnetic field on crystal growth by floating-zone method in microgravity
}

\author{
LI Kai (李 凯) ${ }^{*}$ \& HU Wenrui (W. R. HU, 胡文瑞)
}

National Microgravity Laboratory, Institute of Mechanics, Chinese Academy of Sciences, Beijing 100080, China Correspondence should be addressed to Hu Wenrui (email: wrhu@ mail.imech.ac.cn)

Received November 3, 2000

\begin{abstract}
The magnetic damping effect of the non-uniform magnetic field on the floating-zone crystal growth process in microgravity is studied by numerical simulation. The results show that the non-uniform magnetic field with designed configuration can effectively reduce the flow near the free surface and then in the melt zone. At the same time, the designed magnetic field can improve the impurity concentration non-uniformity along the solidification interface. The primary principles of the magnetic field configuration design are also discussed.
\end{abstract}

Keywords: floating-zone method, crystal growth, non-uniform magnetic field, numerical simulation, microgravity.

The floating-zone method is to grow crucible-free crystal in such a process that the contamination of the melt and the restriction on the melting temperature of the grown crystal by the crucible material can be avoided. However, the diameter of the grown crystal is limited by the normal gravity under the terrestrial condition. The microgravity environment provides the possibility of growing large size crystal by the floating-zone method, and the largest grown crystal is $50 \mathrm{~mm}$ in diameter at present ${ }^{[1]}$. In this case, the thermocapillary convection driven by the surface tension gradient is dominant in the melt zone. The thermocapillary convection may be unsteady, and induce the impurity striations in the grown crystal, especially for the crystal with small Pr number. So a longitudinal steady magnetic field is often used to suppress the unsteady convection in the melt ${ }^{[2-4]}$, and then eliminate the impurity striations in the corresponding part of the grown crystal $^{[4]}$. However, the longitudinal magnetic field has no direct influence on the flow velocity along the force-lines and the flow near the free surface is still relatively strong. Such a flow structure will induce large non-uniformity in concentration distribution in the melt zone, particularly at the solidification interface, which results in the large radial macro-segregation in the grown crystal. So it is important to improve the concentration distribution non-uniformity at the solidification interface by a suitable flow structure in the melt zone while suppressing the unsteady convection by using the applied magnetic field.

The magnetic field has two freedoms: strength and configuration. For the longitudinal magnetic field, only the field strength is adjustable, and the above goal cannot be achieved by merely increasing the field strength $^{[2,5]}$. So the application of the non-uniform magnetic field whose strength and configuration can be adjusted simultaneously is particularly important for the floating-

* Present address: School of Mechanical and Materials Eng., Washington State University, Pullman, WA 99164-2920, USA. 
zone crystal growth process in micogravity. However, the study on this subject has not been fully developed $^{[6-8]}$. In the present paper, the effect of non-uniform magnetic field configuration on the convection, temperature and concentration distributions for P-doped $\mathrm{Si}$ growth by floating-zone method in microgravity is studied by numerical simulation, and the principles of the non-uniform magnetic field design are also studied.

\section{Numerical model and method}

The model of the floating-zone method is described in a cylindrical coordinate system ( $r$, $\theta, z)$ and is assumed to be axis-symmetric, $\partial / \partial \theta=0$ as shown in fig. 1. A cylinder liquid bridge is floating between the phase change interfaces at $z=0$ and $z=L$ respectively, with the same radius $R_{0}$ under microgravity condition. The phase change interfaces are assumed to be flat with constant melting temperature $T_{0}$ respectively. The dependence of the interface melting temperature on the impurity concentration is not included because the considered impurity concentration is dilute. Gaussian ambient Fig. 1 . The schematic diagram of a floating-zone crystal temperature distribution is adopted:

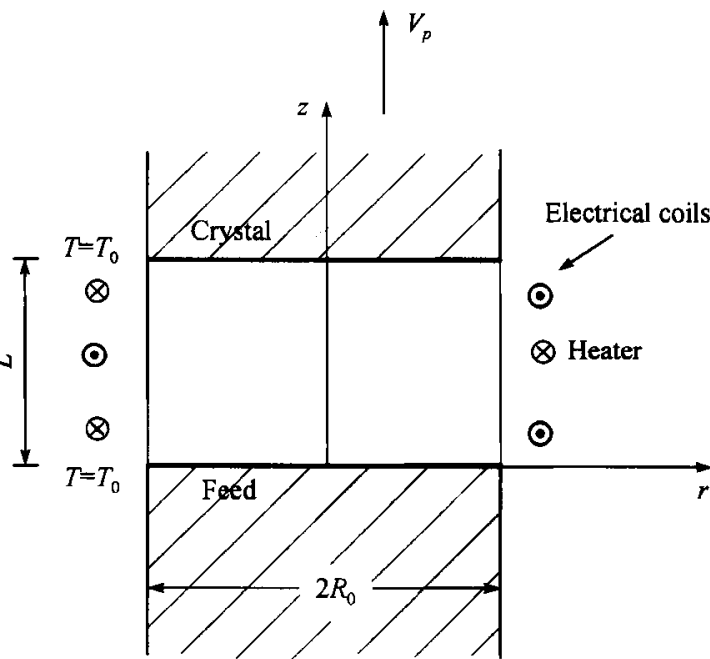

$$
T_{a}(z)=T_{0}+\Delta T \exp \left\{-\left[\left(z-\frac{1}{2} L\right) / a\right]^{2}\right\},
$$

where $\Delta T$ is the temperature difference between the heater and melting point, and $a$ is the width of the distribution. The growth velocity $V_{p}$ is upward in $z$-direction. The designed non-uniform magnetic fields are produced by several electrical coils, which are located axis-symmetrically at adjusted positions and close to the free surface. The diameter of the coils is small and assumed to be zero for simplification. The magnetic fields produced by the electric currents of the coils are computed based on the Biot-Savart's law. Because the magnetic Reynolds number is very small for semiconductors, the induced magnetic field can be neglected. The magnetic field can be shaped by the adjustments of the directions of electric currents, the numbers and the longitudinal positions of electrical coils .

The non-dimensional quantities and parameters are defined as follows :

$$
\begin{aligned}
& r^{*}=\frac{r}{R_{0}}, z^{*}=\frac{z}{R_{0}}, u^{*}=\frac{u}{U_{0}}, v^{*}=\frac{v}{U_{0}}, \quad p^{*}=\frac{p}{\rho U_{0}^{2}}, \\
& T^{*}=\frac{T-T_{0}}{\Delta T}, \quad c^{*}=\frac{c}{c_{0}}, \quad B^{*}=\frac{B}{B_{0}}, \quad V_{p}^{*}=\frac{V_{p}}{U_{0}}, \quad T_{0}^{*}=\frac{T_{0}}{\Delta T}, \\
& R e=\frac{U_{0} R_{0}}{\nu}, \quad M a=\frac{U_{0} R_{0}}{\kappa}, \quad P e=\frac{U_{0} R_{0}}{D}, \quad \Gamma=\frac{L}{2 R_{0}}, \quad B i=\frac{h R_{0}}{k}, \\
& R a=\frac{\varepsilon \sigma R_{0} T_{0}^{3}}{k}, \quad H a=B_{0} R_{0} \sqrt{\sigma_{e} / \rho \nu}, U_{0}=\left|\sigma_{T}\right| \Delta T / \rho \nu, B_{0}=\mu_{r} \mu_{0} I_{0} / \pi R_{0} .
\end{aligned}
$$

The symbols and the physical properties of $\mathrm{P}$-doped $\mathrm{Si}$ are given in the appendix. The superscript * " denotes the non-dimensional quantities and will be omitted hereafter.

The thermocapillary convection in the melt zone is assumed to be steady and axis-symmet- 
ric. The non-dimensional governing equations are written as follows:

$$
\begin{aligned}
& \frac{1}{r} \frac{\partial}{\partial r}(r u)+\frac{\partial}{\partial z}(v)=0, \\
& \frac{1}{r} \frac{\partial}{\partial r}\left(r u^{2}\right)+\frac{\partial}{\partial z}(v u)=-\frac{\partial p}{\partial r}+\frac{1}{R e}\left[\frac{1}{r} \frac{\partial}{\partial r}\left(r \frac{\partial u}{\partial r}\right)+\frac{\partial^{2} u}{\partial z^{2}}-\frac{u}{r^{2}}\right]+\frac{H a^{2}}{R e}\left(v B_{r} B_{z}-u B_{z}^{2}\right), \\
& \frac{1}{r} \frac{\partial}{\partial r}(r u v)+\frac{\partial}{\partial z}\left(v^{2}\right)=-\frac{\partial p}{\partial z}+\frac{1}{R e}\left[\frac{1}{r} \frac{\partial}{\partial r}\left(r \frac{\partial v}{\partial r}\right)+\frac{\partial^{2} v}{\partial z^{2}}\right]+\frac{H a^{2}}{R e}\left(u B_{r} B_{z}-v B_{r}^{2}\right), \\
& \frac{1}{r} \frac{\partial}{\partial r}(r u T)+\frac{\partial}{\partial z}(v T)=\frac{1}{M a}\left[\frac{1}{r} \frac{\partial}{\partial r}\left(r \frac{\partial T}{\partial r}\right)+\frac{\partial^{2} T}{\partial z^{2}}\right], \\
& \frac{1}{r} \frac{\partial}{\partial r}(r u c)+\frac{\partial}{\partial z}(v c)=\frac{1}{P e}\left[\frac{1}{r} \frac{\partial}{\partial r}\left(r \frac{\partial c}{\partial r}\right)+\frac{\partial^{2} c}{\partial z^{2}}\right],
\end{aligned}
$$

the gravity term in (1.5) is omitted under the microgravity condition.

The non-dimensional boundary conditions are written as

$$
\begin{aligned}
& r=0, z \in(0, \Gamma): u=0, \frac{\partial v}{\partial r}=0, \frac{\partial T}{\partial r}=0, \frac{\partial c}{\partial r}=0, \\
& r=1, z \in(0, \Gamma): u=0, \frac{\partial v}{\partial r}=-\frac{\partial T}{\partial z}, \\
& -\frac{\partial T}{\partial r}=B i\left(T-T_{a}(z)\right)+R a\left[\left(T+T_{0}\right)^{4}-\left(T_{0}+T_{a}(z)\right)^{4}\right], \frac{\partial c}{\partial r}=0, \\
& z=0, r \in(0,1): u=0, v=V_{p}, T=0, \frac{\partial c}{\partial z}=V_{p} P e(c-1), \\
& z=\Gamma, r \in(0,1): u=0, v=V_{p}, T=0, \frac{\partial c}{\partial z}=V_{p} P e\left(1-k_{0}\right) c,
\end{aligned}
$$

where $k_{0}$ is the impurity segregation coefficient and is assumed to be a constant .

The steady governing equations are treated as "pseudo-unsteady" equations and discretized on the staggered grids by the finite control volume difference method. Fully implicit time marching is used with first order in time term. Hybrid scheme is adopted in writing the coefficients of convection and diffusion term. The "block modification" method ${ }^{[9]}$ is used to solve the problem of difference equations. The boundary conditions are discretized with second order accuracy. The total grid meshes are $32 \times 64$. The variable convergence criteria may be estimated as follows:

$$
\begin{aligned}
\left|\frac{u-u_{\text {old }}}{u_{\text {old }}}\right|_{\max }<1.0 \times 10^{-5},\left|\frac{v-v_{\text {old }}}{v_{\text {old }}}\right|_{\max }<1.0 \times 10^{-5}, \\
\left|\frac{T-T_{\text {old }}}{T_{\text {old }}}\right|_{\max }<1.0 \times 10^{-5},\left|\frac{c-c_{\text {old }}}{c_{\text {old }}}\right|_{\max }<1.0 \times 10^{-5},
\end{aligned}
$$

where "old" denotes the previous iteration and "max" means the maximum value of the absolute value. The residual of each governing equation is smaller than $1.0 \times 10^{-7}$.

\section{Numerical simulation results}

According to previous study ${ }^{[6]}$, a uniform longitudinal magnetic field which is produced approximately by four electrical coils with $I_{0}=4.0 \times 10^{4} \mathrm{~A}$ in the same direction in each coil is given in the present paper (fig. 2(e)), and the maximum magnetic field strength in the melt zone is $0.988 \mathrm{~T}$. It may be practically difficult to carry the large electric currents in such small electrical coils, but in the present study, such coil design is simply used to generate the non-uniform mag- 
netic field with the required configuration and strength. It can be seen that for the floating-zone crystal growth process in microgravity, the thermocapillary convection in the melt zone is driven by the gradient of the free surface tension, and the flow intensity near the free surface is the strongest. It is also the poor damping effect of the longitudinal magnetic field on the strong flow along the free surface that results in the non-uniformity of the residual flow field. So the non-uniform magnetic fields are designed specially to reduce the flow near the free surface and then in the melt zone with the reduction of the convection driven force by the local magnetic damping effect. At the same time, the non-uniformity of the residual flow field can be improved.

The non-uniform magnetic fields produced by two coils are studied at first. It brings fundamental insights into the principles of the non-uniform magnetic field design. When each coil carries an equal electric current $I_{0}=2.0 \times 10^{3} \mathrm{~A}$ in the opposite direction compared with its neighbour coil, a horizontal plane of purely radial magnetic field appears in between the coils. The coil distance in previous study ${ }^{[8]}$ is adopted in the present paper. Such configuration has the characteristic that only the designed field at the purely horizontal plane of the radial magnetic field near the free surface has similar field strength in comparison with the longitudinal magnetic field, while the field strength is much weaker in other regions ( fig. 2). When the horizontal plane of purely radial magnetic field is located around the point which relates to the maximum flow velocity on the upper half part of the free surface in the case without magnetic filed (fig. 2(a)), the magnetic damping effects on the flow field, temperature field and concentration field are investigated in fig. 3 (a). The strong radial magnetic field component greatly reduced the flow velocity on the upper half of the free surface and then the flow in the upper half zone. Moreover, the relatively uniform residual flow velocity on the upper half of the free surface resulted in the more uniform residual flow field near the solidification interface and then the radial concentration distribution nonuniformity at the solidification interface is improved greatly. The flow in the lower half zone is not suppressed obviously because of the weak magnetic field there. The strong convective circulation even developed into a part of the upper half zone. It also verified the conclusion that the flow in the melt zone could be damped effectively by the reduction of the flow velocity on the free surface. However, the maximum longitudinal velocity and the maximum radial velocity in the melt zone are only reduced by about $43 \%$ and $30 \%$ respectively in comparison with the case without magnetic field because of the strong flow in the lower half zone. When the horizontal plane of purely radial magnetic field is located around the point which relates to the maximum flow velocity

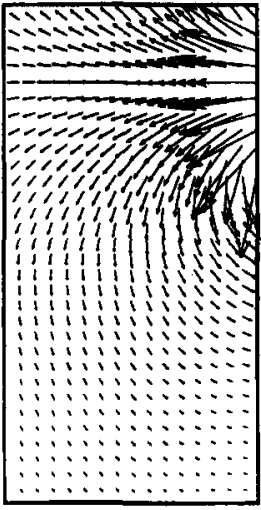

(a)

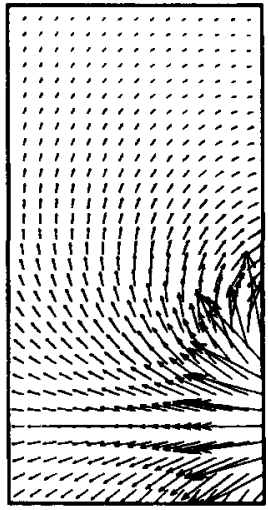

(b)

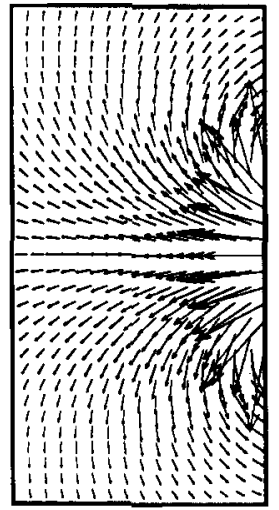

(c)

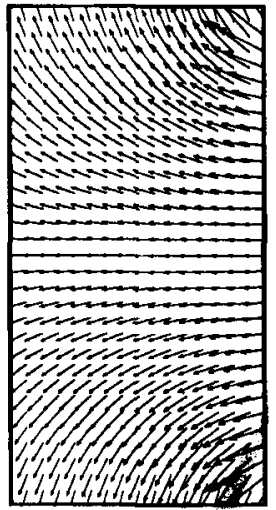

(d)

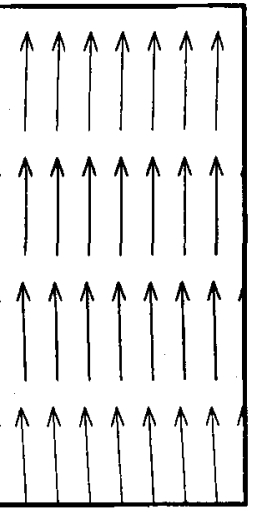

(e)

Fig. 2. The two-coil magnetic field configurations when the coils are located at: (a) $z=2.0, z=1.4 ;(b) z=0.6$, $z=0.0 ;$ (c) $z=1.3, z=0.7 ;$ (d) $z=2.0, z=0.0$ respectively and (e) the longitudinal magnetic field. 

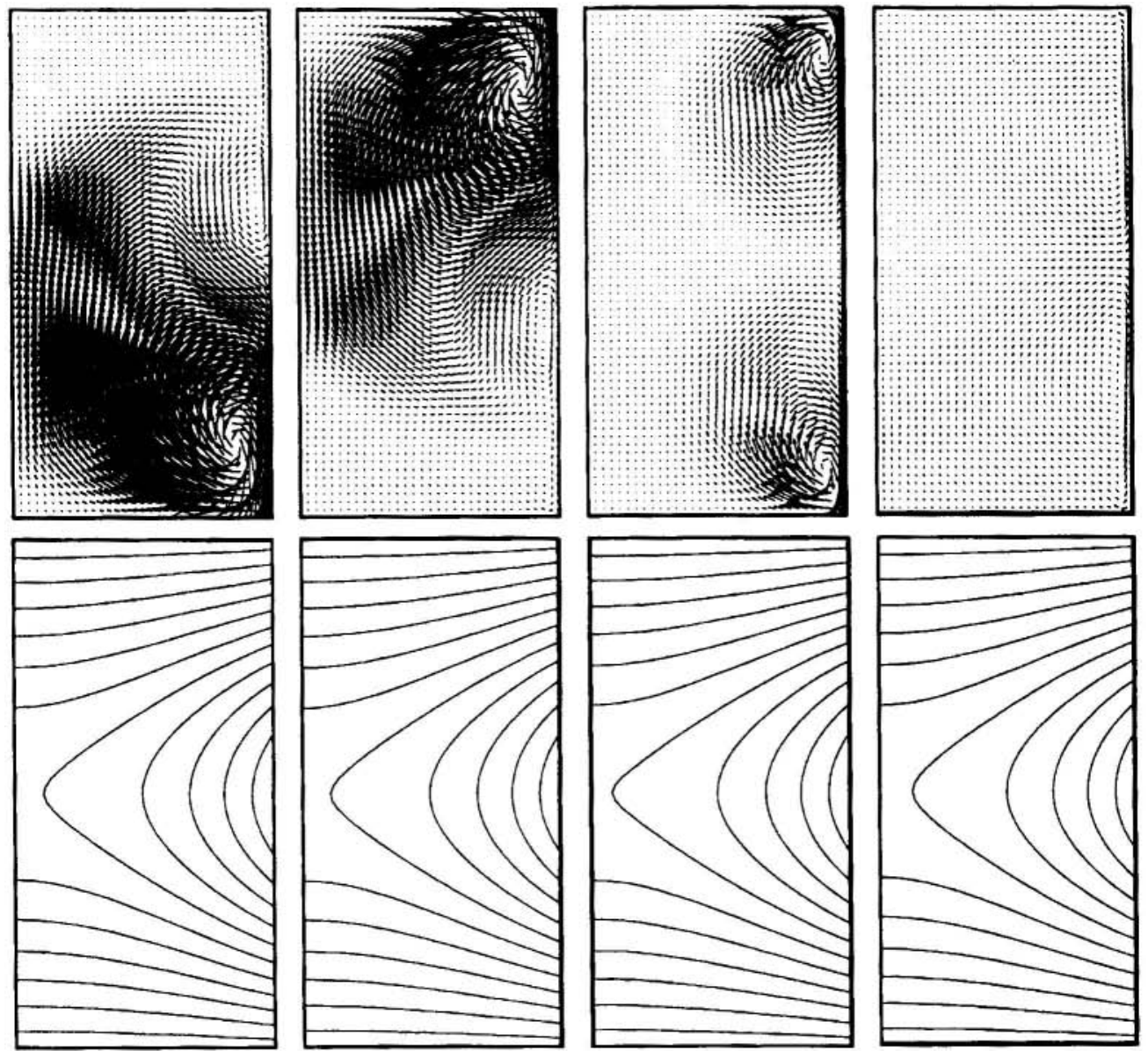

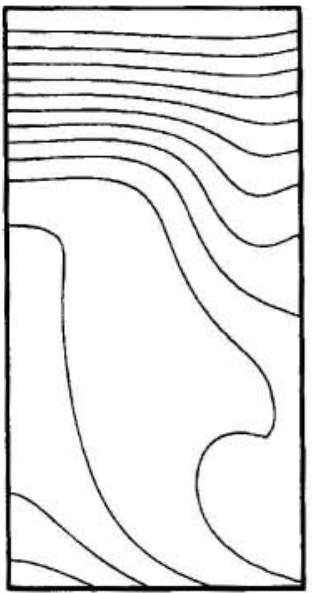

(a)

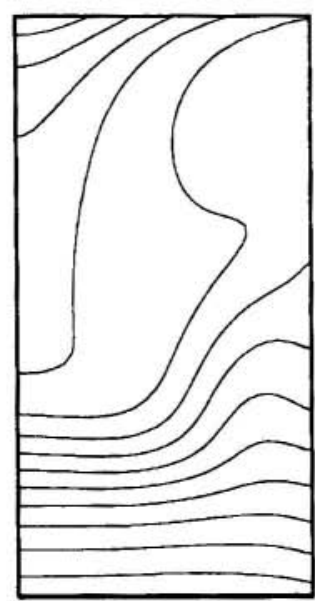

(b)

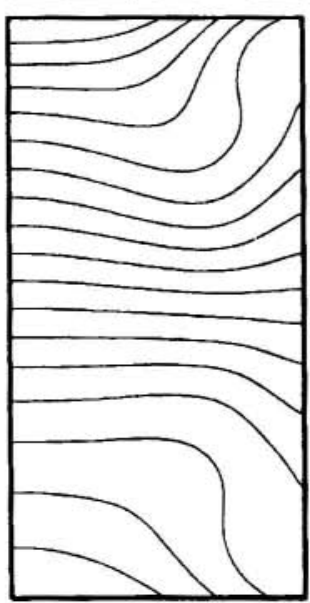

(c)

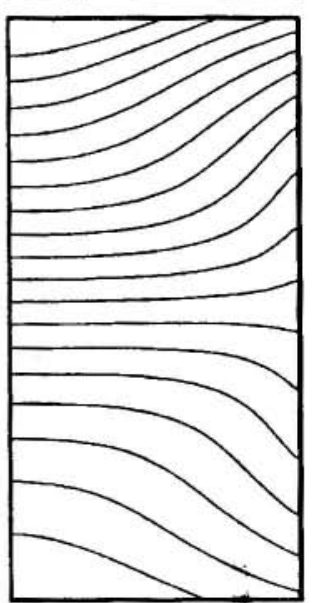

(d)

Fig. 3. The flow fields (upper), temperature fields (middle) and concentration fields (lower) in the caees of two-coil magnetic fields when the coils are located at: (a) $z=2.0, z=1.4$; (b) $z=0.6, z=0.0 ;(c) z=$ $1.3, z=0.7$; (d) $z=2.0, z=0.0$ respectively. 
on the lower half part of the free surface in the case without magnetic field (fig. $2(\mathrm{~b})$ ), the damped flow field, temperature field and concentration field are almost symmetric to those of the case shown in fig. 3(a) according to middle cross section of the melt zone (fig. 3(b)). However, the radial concentration distribution non-uniformity at the solidification interface is increased because of the strong flow in the upper half zone. When the horizontal plane of purely radial magnetic field is located around the middle point of the free surface (fig. 2(c)), the strong radial magnetic field component distribution on the free surface is greatly increased. Thus the flow in the whole melt zone is reduced effectively and the maximum longitudinal velocity and the maximum radial velocity in the melt zone are reduced by about $78 \%$ and $62 \%$ respectively in comparison with the case without magnetic field (fig. 3(c)). However, the magnetic damping effect at the center of the free surface is stronger than that at two ends of the free surface, and the flow field structure uniformity near the solidification interface is damaged by the relatively strong flow at the up right corner of the melt zone. It induces even larger impurity concentration distribution nonuniformity along the solidification interface. The concentration distributions at the solidification interface of the above cases are illustrated in fig. 4 , and the concentration distribution non-uniformity is defined as

$$
\zeta=\frac{c_{\max }-c_{\min }}{\bar{c}},
$$

where $c_{\max }, c_{\min }$ and $\bar{c}$ are maximum, minimum and average concentration values at the solidification interface respectively. Moreover, the magnetic damping effect on the temperature distribution in the crystal growth process of small Pr number is not obvious. From the study above, it is effective to reduce the flow near the free surface and then the flow in the melt zone by the designed non-uniform magnetic field. The flow near the solidification interface has direct and strong influence on the impurity transfer along the solidification interface, and the uniform flow structure helps improve the concentration distribution uniformity along the solidification interface. Moreover, the relatively uniform flow structure near the

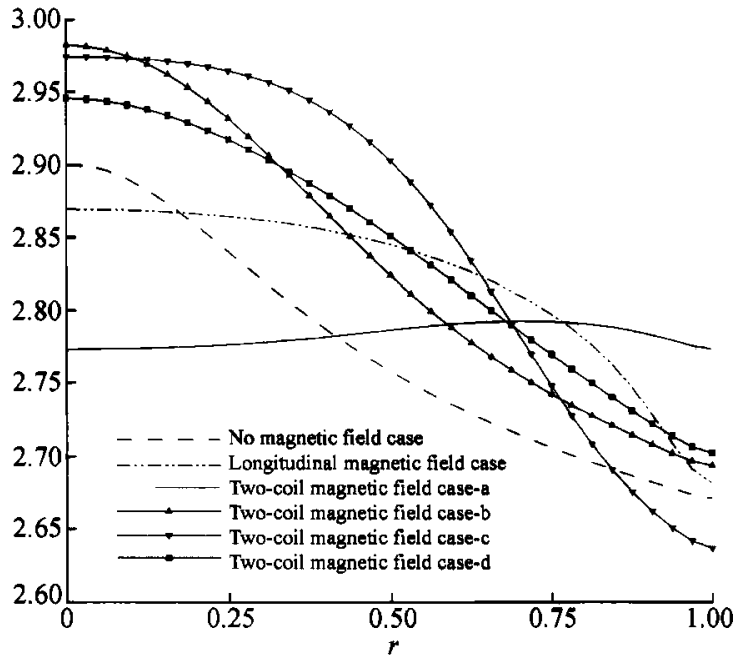

Fig. 4. The profiles of the concentration distribution at the solidification interface in the cases of two-coil magnetic field when the coils are located at: $a, z=2.0, z=1.4, \zeta=0.7 \% ; b, z$ $=0.6, z=0.0, \zeta=10.2 \% ; c, z=1.3, z=0.7, \zeta=$ $11.8 \% ; \mathrm{d}, z=2.0, z=0.0, \zeta=8.6 \%$ respectively in comparison with the case without the magnetic field $(\zeta=8.3 \%)$ and solidification interface is available by the re- with the longitudinal magnetic field $(\zeta=6.7 \%)$.
duction of flow in the upper half zone by the designed non-uniform magnetic field.

In the study above, the strong radial magnetic field component distribution on the free surface is limited, and the flow in some parts of the melt zone is still relatively strong. In order to suppress the unsteady convection, the strong radial magnetic field component distribution on the free surface should be increased as much as possible to reduce the relatively strong flow, and then the maximum flow velocity in the whole melt zone. So based on fig. 2 (c), with increasing distribution of the strong radial magnetic field component on the free surface (fig. 2(d)), the reduction of the flow velocity on the free surface increases, so does the flow at the corner of the melt zone near the free surface (fig. 3(d)). Then the maximum flow velocity in the whole melt zone 
decreases, the maximum longitudinal and radial velocities are reduced by about $98 \%$ and $84 \%$ respectively, and help to suppress the unsteady convection in the melt zone in comparison with the case without magnetic field. On the other hand, the poor impurity mixture by the weak flow in the melt zone results in even larger impurity concentration non-uniformity along the solidification interface (fig. 4) in comparison with the two-coil case shown in fig. 3(a). So the flow with certain intensity in the melt zone is beneficial to the impurity mixture, and then the improvement of the impurity concentration non-uniformity along the solidification interface.

From the study above, it can be seen that in order to suppress the unsteady convection, the flow intensity in the melt zone should be reduced by the applied magnetic field as much as possible. On the other hand, the uniform residual flow structure near the solidification interface and the good impurity mixture by the flow with certain intensity in the melt zone help to improve the impurity concentration non-uniformity along the solidification interface. So there must be a good compromise between the two sides by using the applied magnetic field unless the crystal growth is a purely diffusion process. As the practice of above principles of the magnetic field design, a non-uniform magnetic field produced by four electrical coils is applied in the melt zone (fig. 5 (a) ) based on the case shown in fig. 2(a). The upper and lower pairs of coils are located symmetrically according to the middle cross section of the melt zone, and its magnetic damping effects on the flow field, temperature field and concentration field are shown in fig. 5. The maximum longitudinal and radial velocities are reduced by about $97 \%$ and $86 \%$ respectively, and the impurity concentration non-uniformity along the solidification interface is reduced by about $70 \%$ and $63 \%$ in comparison with the case without magnetic field and with longitudinal magnetic field respectively (fig. 6). It can be seen that the non-uniform magnetic field with designed configuration can reduce the flow in the melt zone and improve the impurity concentration non-uniformity along the solidification interface at the same time.

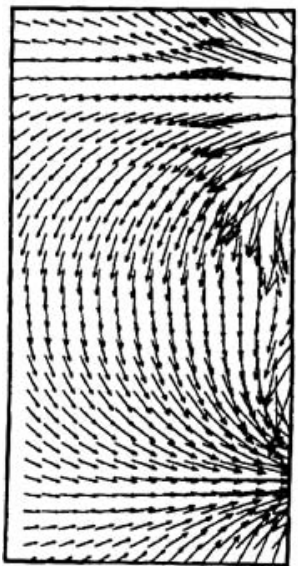

(a)

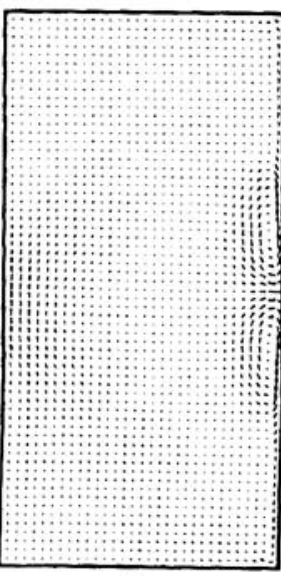

(b)

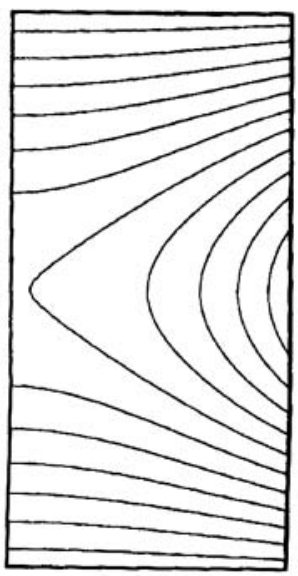

(c)

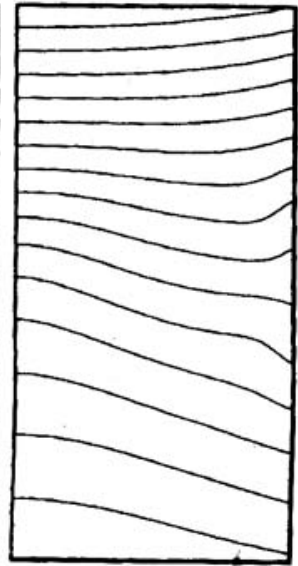

(d)

Fig. 5. The magnetic field configuration (a), the flow field (b), temperature field (c) and ancentration field $(d)$ in the case of the designed magnetic field produced by four coils. $\Delta T^{2} \Delta x$ are re spectively the same as those in fig. 3 .

\section{Conclusion}

For the floating-zone crystal growth process in microgravity, the thermocapillary convection driven by the free surface tension gradient is dominant in the melt zone. In order to suppress the unsteady convection, the non-uniform magnetic field with designed configuration can effectively 
reduce the flow near the free surface and then in the melt zone. At the same time, the designed magnetic field can improve the impurity concentration non-uniformity along the solidification interface. It should be noted that further study on the effect of the phase change interface shape on the impurity concentration distribution to improve the simplified model to relate the practice is necessary .

\section{Appendix}

$(u, 0, v)$, velocity vector; $\boldsymbol{B}\left(B_{r}, 0\right.$, $B_{z}$ ), magnetic field vector; $\Gamma=1.0$, aspect ratio of the melt zone; $\kappa=0.255 \mathrm{~cm}^{2} /$ $\mathrm{s}$, thermal diffusion coefficient; $\nu=0.0035$ $\mathrm{cm}^{2} / \mathrm{s}$, kinematic viscosity; $\sigma_{e}=1.23 \times$ $10^{6} / \Omega^{-1} \mathrm{~m}^{-1}$, electrical conductivity; $\mu_{r}=$

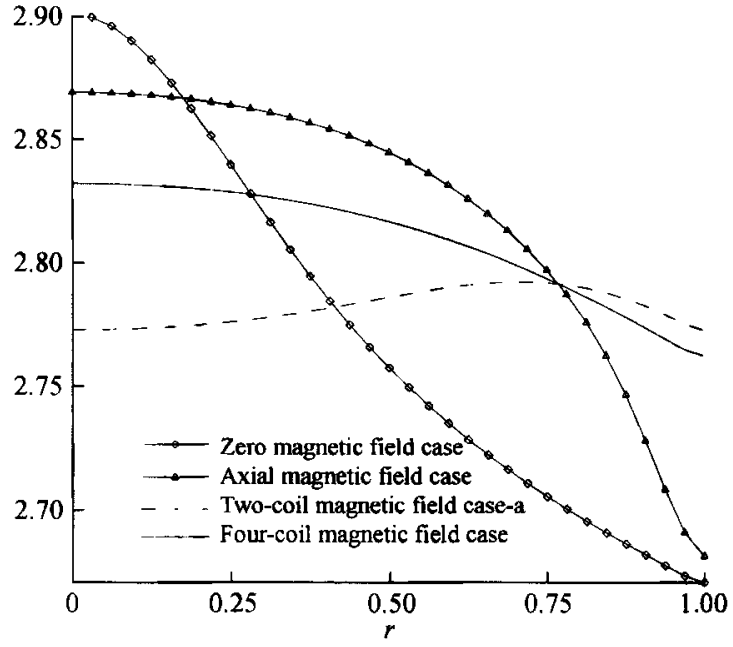

Fig. 6. The profiles of the concentration distribution at the solidification interface in the cases without magnetic field, with the longitudinal magnetic field, with the two-coil magnetic field and with the four-coil magnetic field respectively. The non-uniformity which is defined as $\zeta$ is $8.3 \%, 6.7 \%, 0.7 \%$ and $2.5 \%$ respectively. 1.0 , relative magnetic permeability; $T_{0}=1683 \mathrm{~K}$, melt point; $\rho=2.53 \mathrm{~g} / \mathrm{cm}^{3}$, density; $k=$ $0.67 \mathrm{~W} / \mathrm{cmK}$, thermal conductivity; $k_{0}=0.35$, impurity segregation coefficient; $\varepsilon=0.3$, emissivity; $V_{p}=5.0 \times 10^{-6} \mathrm{~m} / \mathrm{s}$, growth rate; $\mu_{0}$, magnetic permeability in vacuum; $c_{0}$, impurity concentration in the feed; $h$, heat transfer coefficient; $\sigma$, Stefan-Boltzmann constant; $I_{0}$, electric current in the coil; $U_{0}$, characteristic velocity; $B_{0}$, characteristic magnetic field; $\Delta T=$ $10 \mathrm{~K}$, temperature difference; $D=5.0 \times 10^{-8} \mathrm{~cm}^{2} / \mathrm{s}$, impurity diffusion coefficient; $\sigma_{\mathrm{T}}=$ $-1.3 \times 10^{-4} \mathrm{~N} / \mathrm{mK}$, gradient of surface tension.

Acknowledgements This work was supported by the Project 95-yu-34 of the Ministry of Science and Technology of China and the National Natural Science Foundation of China (Grant No. 19789201).

\section{References}

1. Markov, E. V., Antropov, V. Yu, Biryukov, V. M. et al., Space materials for microelectronics, in Proceedings of the Joint Xth European and VIth Russian Symposium on Physical Sciences in Microgravity, St. Petersburg, Russia (eds. Avduyevsky, V. S., Polezhaev, V. I.), Moscow: RAS, 1997, Vol. 2, 11-20.

2. Croll, A., Dold, P., Benz, K. W., Segregation in Si floating-zone crystals grown under microgravity and in a magnetic field, J. Crystal Growth, 1994, 137: 95-101.

3. Leon de, N., Guldberg, J., Salling, J., Growth of homogeneous high resistivity FZ silicon crystals under magnetic field bias, J. Crystal Growth, 1981, 55: 406-408.

4. Robertson, D. G., O' connor Jr., D. J., Magnetic field effects on float-zone Si crystal growth : strong axial fields, J. Crystal Growth, 1986, 76: 111-122.

5. Series, R. W., Hurle, D. T. J., The use of magnetic field in semiconductor crystal growth, J. Crystal Growth, 1991, 113: $305-328$.

6. Lan, C. W. , Effect of axisymmetric magnetic fields on radial dopant segregation of floating-zone silicon growth in a mirror furnace, J. Crystal Growth, 1996, 169: 269-278.

7. $\mathrm{Li}, \mathrm{K} ., \mathrm{Hu}, \mathrm{W} . \mathrm{R}$., Numerical simulation of magnetic field design for damping thermocapillary convection in a floating half zone, J. Crystal Growth, 2001, 222: 677-684.

8. Li, K., Hu, W. R., Magnetic design for crystal growth, 3rd International Workshop on Modeling in Crystal Growth, New York, USA, 2000, to be published in J. Crystal Growth.

9. Patankar, S. V., Advanced Computational Heat Transfer and Fluid Flow, Graduate Student Course 8352 of Mechanical Engineering Department at Univ. of Minnesota, USA. 